\title{
COLLISIONAL REMOVAL OF HI FROM THE INNER DISKS \\ OF VIRGO CLUSTER GALAXIES
}

\author{
MONICA VALLURI and CHANDA J. JOG \\ Department of Physics, Indian Institute of Science, \\ Bangalore, INDIA
}

\begin{abstract}
In addition to the well established global HI-deficiency seen in Virgo cluster spirals, many of them are found to be HI-deficient in their inner disks. We show that collisions between galaxies in a cluster can lead to the preferential removal of HI from these galaxies. This follows directly from the application of the Spitzer-Baade collisional gas removal mechanism to galaxies consisting of stars and a two-component ISM of $\mathrm{HI}$ and $\mathrm{H}_{2}$, with $\mathrm{HI}$ having the largest filling factor. This can account for both the observed HI-deficiency in the inner disks of these galaxies as well as their normal $\mathrm{H}_{2}$ contents. The frequency of galaxy collisions in the Virgo cluster is shown to be large enough to make collisional gas removal a viable mechanism.
\end{abstract}

\section{INTRODUCTION}

The global HI-deficiency in spiral galaxies in clusters is largely due to the loss of HI gas from their outer disks (for a review see Haynes, Giovanelli, and Chincarini 1984). It is believed that gas is removed from galaxies by ram-pressure stripping by the intracluster medium (ICM), (Gunn and Gott 1972), or during collisions between galaxies (Spitzer and Baade 1951) or by other mechanisms involving galaxy-ICM interactions. Using the HI data from Warmels (1986), for spiral galaxies in the Virgo cluster, we show that a large fraction of galaxies are not only deficient globally but also in their inner disk regions.

CO observation of HI-deficient galaxies in Virgo, (e.g. Kenney and Young 1989), have shown that the $\mathrm{H}_{2}$ component in these galaxies has been unaffected by the removal of $\mathrm{HI}$.

Ram-pressure stripping, the most popular gas removal mechanism, is expected to be less effective in removing $H I$ from the inner disks, $\left(r<R_{0} / 2\right.$, with $R_{0}$ being the Holmberg radius $)$, where the gravitational binding force is higher than it is at larger radii and where, in addition, the $\mathrm{H}_{2}$ gas is concentrated. We propose that collisions between galaxies is an important mechanism by which $H I$ can be removed from their inner disks while leaving the $\mathrm{H}_{2}$ component undisturbed.

\section{HI DEFICIENCY IN THE INNER DISKS}

From high resolution mapping of spiral galaxies both in the Virgo cluster and in the field, Warmels (1986) has obtained estimates for the degree of HI-deficiency in the inner disks of cluster galaxies. The degree of deficiency of a cluster galaxy is estimated by the parameter 


$$
\mathrm{DEF}_{\mathrm{IN}}=\log \left[\frac{<\sigma_{\mathrm{HI}}>_{\mathrm{T}, \text { field }}}{\left(\sigma_{\mathrm{HI}}\right)_{\mathrm{T}, \text { cluster }}}\right]
$$

where $\left(\sigma_{\mathrm{HI}}\right)_{\mathrm{T}}$, cluster is the $\mathrm{HI}$ surface density averaged over half the Holmberg radius $\left(\mathrm{R}_{0} / 2\right)$ for a cluster galaxy, and $<\sigma_{\mathrm{HI}}>_{\mathrm{T}}$,field is the mean value of $\sigma_{\mathrm{HI}}$ for a sample of field galaxies of the same morphological type T. A galaxy is usually considered HI-deficient if it has a factor of two less $\mathrm{HI}$ than an average field galaxy (i.e., $\mathrm{DEF}_{\mathrm{IN}} \geq 0.3$ ). We show that, of the 26 galaxies in Warmels' sample lying within the Abell radius ( $5^{\circ}$ from M87), $22 \%$ are HI-deficient in their inner disks, this is much greater than the $7 \%$ expected to have such a low value of $\sigma_{\mathrm{HI}}$ purely from the gaussian spread in this quantity. Kenney and Young (1989) have shown from the same data that galaxies that are globally deficient in HI by a factor of 10 , are HI-deficient in their inner disks by a factor of 2-3.

\section{PHYSICAL MECHANISM FOR COLLISIONAL GAS REMOVAL}

Spitzer and Baade (1951) showed that when cluster galaxies collide with a high relative velocity $\left(\mathrm{V}_{\text {rel }} \sim 2000 \mathrm{~km} \mathrm{~s}^{-1}\right.$, their stellar components are unperturbed, but their ISM components with their large filling factors, undergo inelastic collisions. If $V_{\text {rel }}>V_{e s c}$, the escape velocity from the galaxy, (with $\mathrm{V}_{\text {esc }} \sim 300 \mathrm{~km} \mathrm{~s}^{-1}$ ), the colliding gas components would be lost from the galaxies since the kinetic energy of the collision would be thermalised and would heat the gas to a temperature of $\sim 5 \times 10^{7} \mathrm{~K}$, giving it sufficient energy to escape from the disk. Even if the gas cooled rapidly, it would condense as a cold cloud and would be left behind at the center of mass of the receding galaxies.

The Importance Of Filling Factor

Gas clouds of a particular ISM component will collide if their mean free path, $(\lambda)$, is less than the dimensions of the region of overlap of the two galaxies. Spherical clouds of radii, $R_{c}$, of an ISM component of filling factor, $f$, will have a mean free path $(\lambda)$ given by

$$
\lambda=\frac{2 R_{c}}{3 f}
$$

Gas clouds from the two galaxies will collide if $\lambda$ is less than the maximum dimension, $d$, of the overlapping region. The HI component consists of at least three sub-components, with the cold neutral medium of Kulkarni and Heiles (1987) having the smallest filling factor. If we consider the standard Spitzer clouds with $\mathrm{R}_{c}=5 \mathrm{pc}$, and $\mathrm{f}=0.1$, then the mean free path, $\lambda$ $=35$ pc. In the extreme case of a face-on collision, $\mathrm{d}$ is a minimum $\approx 2 \mathrm{Z}_{1 / 2}$, or twice the scale height of the gas distribution. Since $Z_{1 / 2}=100 \mathrm{pc}$ for the clouds of the cold neutral medium, $\lambda \ll \mathrm{d}$, and hence even in this case the HI clouds in the overlapping volume would collide. The other HI components are believed to be in sheet-like or filamentary clouds and to have a larger filling factor. Hence all the HI gas in the overlapping volume will be lost from the colliding galaxies.

For the $\mathrm{H}_{2}$ clouds, on the other hand, $\mathrm{R}_{c}=25 \mathrm{pc}, \mathrm{f}=0.02$, and $\mathrm{Z}_{1 / 2}=65 \mathrm{pc}$, hence from 
eq.(1), $\lambda=1 \mathrm{kpc}$ which is much greater than $\mathrm{d}=0.13 \mathrm{kpc}$. Hence the $\mathrm{H}_{2}$ clouds will rarely collide and are unaffected by the encounter.

Therefore, the filling factor of a particular gas component is critical in determining whether or not it will be stripped from the colliding galaxies. Collisions between galaxies is therefore a mechanism by which HI can be preferentially removed from the inner disk of a galaxy leaving the $\mathrm{H}_{2}$ unaffected (Valluri and Jog 1990a).

\section{Collision Frequency}

To obtain an estimate of the collision frequency we assume that spiral galaxies within the $5^{\circ}$ -region have isotropic orbits and an isotropic velocity disribution. The one-dimensional velocity dispersion of spiral galaxies in this region is $\sim 800 \mathrm{~km} \mathrm{~s}^{-1}$ and their number density, $\mathrm{n} \sim 15$ galaxies $\mathrm{Mpc}^{-3}$. The galaxies in Warmels' sample, (believed to represent the entire spectrum of galaxies in the cluster), are distributed into three bins of logarithmically increasing galaxy size $\left(R_{0}\right)$. The number of galaxies in each bin $\left(\mathrm{N}_{i}\right)$ is almost the same and hence the bin number density $n_{i}=n / 3$. The total collision frequency for a galaxy in the $i^{\text {th }}$ bin with mean radius $<R_{0}>_{i}$ is

$$
\omega_{c i}=2 \pi \frac{n}{3} V_{r e l} \sum_{j=1}^{3}\left[\frac{\left\langle R_{0}\right\rangle_{i}+\left\langle R_{0}\right\rangle_{j}}{2}\right]^{2}
$$

If galaxies have been undergoing collisions for $1 / 2$ the Hubble time $\left(\mathrm{t} \sim 6 \times 10^{9} \mathrm{yr}\right)$, the collision probability $\mathrm{P}_{c i}=\omega_{c i} \cdot 6 \times 10^{9}$. In Table 1 we compare the fraction $\left(\mathrm{F}_{D}\right)$ of deficient galaxies in each bin with the corresponding collision probability $\mathrm{P}_{c i}$.

\section{TABLE 1.}

COMPARISON OF COLLISION PROBABILITY WITH DEFICIENT FRACTION

\begin{tabular}{cccccr}
\hline $\mathrm{i}$ & SIZE* & MEAN & NUMBER OF & COLLISION & DEFICIENT \\
& RANGE & SIZE & GALAXIES & PROBABILITY & FRACTION \\
& $<R_{0}>$ & $<R_{0}>_{i}$ & $\mathrm{~N}_{\boldsymbol{i}}$ & $\mathrm{P}_{c}$ & $\mathrm{~F}_{D}$ \\
\hline 1 & $<7.0 \mathrm{kpc}$ & $5 \pm 1$ & 8 & $0.06 \pm 0.02$ & $0.13(1 / 8)$ \\
2 & $7.0-11.0$ & $9 \pm 1$ & 9 & $0.09 \pm 0.03$ & $0.22 \pm(2 / 9)$ \\
3 & $>11.0 \mathrm{kpc}$ & $15 \pm 2$ & 9 & $0.15 \pm 0.05$ & $0.33(3 / 9)$ \\
\hline * Holmberg radius & & & & \\
\hline
\end{tabular}

We note that both $P_{c}$ and $F_{D}$ increase with galaxy size and in each case $P_{c} \sim 1 / 2 \cdot F_{D}$. The value of $P_{c}$ may be uncertain by a factor of few due to the assumption of isotropic orbits and the uncertainty in the number density of galaxies. However the trend of an increase in the fraction of deficient galaxies with increasing size points to collisional gas removal as a likely mechanism rather than ram-pressure stripping. 
In a related work currently in progress, we find that in the three rich clusters A262, A1367, and A1656, the fraction of globally HI-deficient spiral galaxies increases with size, dropping only for the largest size bin. We also find that the most highly deficient galaxies are always of the largest sizes. A much larger sample of 81 galaxies in the Virgo cluster also exhibits a similar trend. This clearly indicates that the dominant gas removal mechanism is more effective on larger galaxies than on smaller galaxies. We find that the collisional gas removal mechanism is the only one that is capable of qualitatively accounting for all the features of the size-dependence of HI-deficiency, (Valluri and Jog 1990b). We conclude, therefore, that collisions between galaxies in a cluster is a mechanism which can result in the preferential removal of the HI component of the ISM from galaxies both globally and from their inner disks.

\section{REFERENCES}

Gunn, J. E., and Gott, J. R. 1972, Ap. J., 176, 1.

Haynes, M. P., Giovanelli, R., and Chincarini, G. 1984, Ann. Rev. Astron. Astrophys., 22, 445.

Kenney, J. D., and Young, J. S. 1989, Ap. J., 344, 71.

Kulkarni, S. R., and Heiles, C. 1987, in Galactic and Extragalactic Astronomy, (2nd ed.), eds. G. Verschuur and K. Kellermann, (New York: Springer Verlag).

Spitzer, L., and Baade, W. 1951, Ap. J., 113, 413.

Valluri, M. and Jog, C. J. 1990a, Ap. J., in press.

Valluri, M. and Jog, C. J. 1990b, in preparation.

Warmels R. 1986, Ph. D. Thesis, Univ. of Gronongen. 